Cognitive function in preschool children after epilepsy surgery. Age of seizure onset and extent of the lesion were predictive of preoperative cognitive development in 50 patients treated surgically at age 3 to 7 years; $70 \%$ were retarded, with IQ $<70$. At 6 months to 10 years after surgery, 11 showed IQ/DQ gains of $>15$ IQ points, but only in children free of seizures. Shorter duration of epilepsy was significantly associated with a postoperative increase in DQ. (Freitag H, Tuxhorn I. Epilepsia April 2005;46:561-567).

\title{
COGNITION AND BEHAVIOR WITH IDIOPATHIC EPILEPSIES
}

Cognition and behavior in 42 mainstream school children with newly diagnosed idiopathic or cryptogenic epilepsy ('epilepsy only') were compared with 30 healthy gendermatched classmate controls in a 3.5-year study at Wilhelmina Children's Hospital, Utrecht, The Netherlands. At 3,12 and 42 month follow-up, children with epilepsy performed less well than controls on measures of learning, memory span for words, attention and behavior. Those with epilepsy who repeated a grade continued to have poor scores in Color Trails tests and made more writing errors (so-called "proactive interference"), whereas control children who repeated caught up with non-repeaters. Children with cryptogenic epilepsy scored more poorly. According to parent reports, children with recurring seizures had less behavioral problems than children in remission. Epilepsy patients in a group showed no deterioration in cognition and behavior over time, whereas individually, performance was impaired. Parental poor adaptation to the diagnosis of epilepsy and prediagnostic learning and behavior problems were associated with a child's impaired neuropsychological and school performance, whereas seizure-control or AED treatment was not a factor. (Oostrom KJ, van Teeseling H, Smeets-Schouten A et al. Three to four years after diagnosis: cognition and behaviour in children with 'epilepsy only'. A prospective, controlled study. Brain July 2005;128:1546-1555). (Respond: KJ Oostrom PhD, University Hospital Vrije Universiteit, Department of Medical Psychology, Children's Section, PO Box 7057, 1007 M B Amsterdam, The Netherlands).

COMMENT. The source of impairments in cognition and behavior in children with epilepsy may be found in parental attitudes and lack of acceptance of the diagnosis rather than antiepileptic drug toxicity or poor seizure control. Prediagnostic learning and behavior problems were also significant factors in continuing school difficulties. Time devoted to parental counseling is important in the management of childhood epilepsy.

A significant psychosocial impact of epilepsy on adolescents is also reported in a UK controlled study; low levels of epilepsy understanding are associated with higher levels of depression and anxiety, and lower self-esteem (Baker GA et al. Epilepsy Behav June 2005;6:556-562).

A survey of behavior problems in children with epilepsy conducted in Canada found that a Child Behavior Checklist demonstrated an increased frequency of elevated behavior scores for all scales, particularly for attention and social problems. Behavior problems found in $40 \%$ of the group were unrelated to the type of epilepsy, EEG or AEDs, but they were significantly correlated with the frequency of learning difficulties, present in $57 \%$. (Keene DL et al. Epilepsy Behav Jun e 2005;6:581-586). 